\title{
Canonical transformations in gauge theories with non-trivial backgrounds
}

\section{Daniele Binosi}

European Centre for Theoretical Studies in Nuclear Physics and Related Area (ECT*)

and Fondazione Bruno Kessler,

Villa Tambosi, Strada delle Tabarelle 286, I-38123 Villazzano (TN), Italy

E-mail: binosidecstar.eu

\section{Andrea Quadri*}

Dept. of Physics, Univ. di Milano and INFN, Sez. di Milano

via Celora 16, I-20133 Milan, Italy

E-mail: andrea.quadri@mi.infn.it

We show how to implement the background field method by means of canonical transformations and comment on the applications of the method to non-perturbative techniques in non-Abelian gauge theories. We discuss the case of the lattice in some details.

Sixth International Conference on Quarks and Nuclear Physics,

April 16-20, 2012

Ecole Polytechnique, Palaiseau, Paris

\footnotetext{
${ }^{*}$ Speaker.
} 


\section{Introduction}

Quantization of non-Abelian gauge theories around topologically non-trivial background fields plays an important role in understanding their non-perturbative regime. Instanton configurations [1] have been thoroughly investigated since the pioneering work of 't Hooft [2]. Another example is provided by chiral soliton models in effective approaches to low-energy QCD [3].

On the other hand, one can also introduce a background gauge connection as a technical tool for fixing the gauge while retaining explicit (background) gauge invariance. This leads to the socalled background field method (BFM) [4]. In the BFM powerful relations between background and quantum 1-PI amplitudes emerge, which have been widely used in order to simplify computations in many different applications, ranging from perturbative Yang-Mills theory [5] and the Standard Model [6] to gravity and supergravity calculations [7].

The BFM has been formulated on the lattice in [8], where the quantization is carried out around a fixed background $\hat{A}_{\mu}$ with periodic boundary conditions. The approach of [8] is however purely perturbative, since the path-integral is restricted to quantum fluctuations in a sufficiently small neighborhood of $\hat{A}_{\mu}$.

One might then ask whether a fully non-perturbative BFM can be implemented on the lattice. In dealing with such a problem, one has to face several major challenges, and in particular the wellknown Neuberger's 0/0 problem [9], which prevents the definition of a consistent non-perturbative BRST symmetry, thus making unclear how to apply the algebraic procedure, usually adopted for the perturbative implementation of the BFM [10].

This difficulty ultimately originates from the existence of Gribov copies: indeed if one integrates the BRST-invariant measure $d \mu_{s}$ on the gauge fields $A_{\mu}$, the auxiliary Nakanishi-Lautrup multiplier and the ghost and antighost pair over a compact cycle (like a gauge group orbit in a lattice gauge theory), one gets zero, due to the fact that on a cycle the gauge-fixing condition exhibits an even number of solutions, cancelling out pairwise [11]. It is thus natural to expect that in a nonperturbative formulation of the BFM there must be some other mechanism, allowing to control the background dependence around each of the Gribov solutions.

A possible way of addressing such open issues is based on the use of canonical transformations $[12,13]$. Specifically, it turns out that whenever the theory is endowed with a Batalin-Vilkovisky (BV) bracket and satisfies the (extended) Slavnov-Taylor (ST) identity in the presence of the background field $[12,14]$, the dependence of the effective action on the background can be recovered by purely algebraic means via a canonical transformation (i.e., a transformation preserving the BV bracket). In addition, this canonical transformation can be written in an explicit form by a fieldtheoretic generalization of the Lie transform, used in classical analytical mechanics for obtaining finite canonical transformations once their generating functional is known [13].

It is worth stressing that these results are valid whenever the extended ST identity holds true. In particular, no dynamical ghosts are needed. Therefore, if one is able to fix the background gauge on the lattice by minimizing some suitably chosen functional (possibly a natural extension of the Wilson gauge-fixing functional in the standard lattice Landau gauge formulation), one might hope to make some progress toward a non-perturbative formulation of the BFM. We will comment further on this point in Sect. 3, after presenting our main mathematical results in Sect. 2. 
We refer the reader to [13] for an overview of the prospects of implementing the BFM by means of the canonical transformations in other non-perturbative contexts, e.g., in the 2-PI formalism or Schwinger-Dyson equations.

\section{Reconstruction of the background dependence via canonical transformations}

For a SU(N) Yang-Mills theory, the BV bracket is defined as [13] (only left derivative assumed in what follows)

$$
\{X, Y\}=\int \mathrm{d}^{4} x \sum_{\phi}\left[(-1)^{\varepsilon_{\phi}\left(\varepsilon_{X}+1\right)} \frac{\delta X}{\delta \phi} \frac{\delta Y}{\delta \phi^{*}}-(-1)^{\varepsilon_{\phi^{*}}\left(\varepsilon_{X}+1\right)} \frac{\delta X}{\delta \phi^{*}} \frac{\delta Y}{\delta \phi}\right] .
$$

The sum runs over the fields $\phi=\left(A_{\mu}^{a}, c^{a}\right)$ and the corresponding antifields $\phi^{*}=\left(A_{\mu}^{* a}, c^{* a}\right)$, with $\varepsilon_{\phi}$, $\varepsilon_{\phi^{*}}$ and $\varepsilon_{X}$ representing the statistics of the field $\phi$, the antifield $\phi^{*}$ and the functional $X$ respectively.

In a linear covariant background gauge, the dependence on the antighost field $\bar{c}^{a}$ can only happen through the combination $\widetilde{A}_{\mu}^{* a}=A_{\mu}^{* a}+\left(\widehat{\mathscr{D}}_{\mu} \bar{c}\right)^{a}$, as a consequence of the antighost equation $\frac{\delta \Gamma}{\delta \bar{c}^{a}}=-\widehat{\mathscr{D}}_{\mu}^{a b} \frac{\delta \Gamma}{\delta A_{\mu}^{* b}}+\left(\mathscr{D}^{\mu} \Omega_{\mu}\right)^{a}$. $\hat{A}_{\mu}$ denotes the background gauge field and $\Omega_{\mu}$ its BRST partner [10]. $\widehat{\mathscr{D}}_{\mu}$ stands for the covariant derivative with respect to (w.r.t.) the background. One can then define the reduced functional $\tilde{\Gamma}$ [13] by eliminating the Nakanishi-Lautrup-dependent terms (for they only enter at tree-level, as a consequence of the usual equation of motion for the Nakanishi-Lautrup multiplier field). Since we will only deal with the reduced vertex functional in what follows, we will simply denote it by $\Gamma$. It obeys the extended ST identity in the presence of a background [12]:

$$
\int \mathrm{d}^{4} x \Omega_{\mu}^{a}(x) \frac{\delta \Gamma}{\delta \widehat{A}_{\mu}^{a}(x)}=-\frac{1}{2}\{\Gamma, \Gamma\}
$$

If one now takes the derivative of Eq. (2.2) w.r.t. $\Omega_{\mu}^{a}$ and set the latter source equal to zero afterwards, the resulting equation [12]

$$
\left.\frac{\delta \Gamma}{\delta \widehat{A}_{\mu}^{a}(x)}\right|_{\Omega=0}=-\left.\left\{\frac{\delta \Gamma}{\delta \Omega_{\mu}^{a}(x)}, \Gamma\right\}\right|_{\Omega=0},
$$

shows that the derivative of the vertex functional w.r.t. the background field equals the effect of an infinitesimal canonical transformation (w.r.t. the BV bracket) on the vertex functional itself. Then, since the BV bracket does not depend on either $\widehat{A}_{\mu}^{a}$ or $\Omega_{\mu}^{a}$, if one were able to write the finite canonical transformation generated by the fermion $\Psi_{\mu}^{a}(x)=\frac{\delta \Gamma}{\delta \Omega_{\mu}^{a}(x)}$, one would control the full dependence of $\Gamma$ on the background fields; and this would happen not only at the level of the counterterms of $\Gamma$, but rather for the full 1-PI Green's functions, thus giving control even over the non-local dependence on the background.

The problem can be thus stated mathematically as follows: given the field and antifield variables $\phi, \phi^{*}$, which are canonical w.r.t. the BV bracket (2.1), i.e.,

$$
\begin{aligned}
\left\{\phi_{i}(x), \phi_{j}(y)\right\} & =\left\{\phi_{i}^{*}(x), \phi_{j}^{*}(y)\right\}=0 \\
\left\{\phi_{i}(x), \phi_{j}^{*}(y)\right\} & =\delta_{i j} \delta^{4}(y-x),
\end{aligned}
$$


and the background field $\widehat{A}_{\mu}^{a}$, find the canonical mapping

$$
\left(\phi(x), \phi^{*}(x) ; \widehat{A}_{\mu}^{a}(x)\right) \mapsto\left(\Phi(x), \Phi^{*}(x)\right),
$$

to the new field and antifield variables $\Phi$ and $\Phi^{*}$ such that the ST identity (2.3), written in these new variables, is automatically satisfied. This last condition translates into determining the canonical variables $\Phi$ and $\Phi^{*}$ which are also solutions of the two equations

$$
\begin{aligned}
& \frac{\delta \Phi(y)}{\delta \widehat{A}_{\mu}^{a}(x)}=\frac{\delta \Psi_{\mu}^{a}(x)}{\delta \Phi^{*}(y)}=\left\{\Phi(y), \Psi_{\mu}^{a}(x)\right\}, \\
& \frac{\delta \Phi^{*}(y)}{\delta \widehat{A}_{\mu}^{a}(x)}=-\frac{\delta \Psi_{\mu}^{a}(x)}{\delta \Phi(y)}=\left\{\Phi^{*}(y), \Psi_{\mu}^{a}(x)\right\} .
\end{aligned}
$$

One can obtain the vertex functional $\Gamma$ expressed in terms of the canonically transformed variables by means of homotopy techniques [12]. This solution fails to respect the (naively expected) exponentiation pattern, due to the dependence of the generating functional $\Psi_{\mu}^{a}$ on the background field $\widehat{A}_{\mu}^{a}$.

The explicit canonical transformation for the fields and antifields of the theory can be explicitly worked out as follows. We introduce the operator [13]

$$
\Delta_{\Psi_{\mu}^{a}(x)}=\left\{\cdot, \Psi_{\mu}^{a}(x)\right\}+\frac{\delta}{\delta \widehat{A}_{\mu}^{a}(x)},
$$

where the first term above represents a (graded) generalization (to the BV bracket and a fermionic generator) of the classical Lie derivative w.r.t a (bosonic) generator (in which case the bracket would be the usual Poisson bracket), while the second term takes into account the above observation on the exponentiation failure.

Next, using the properties of the BV bracket, one can establish the following relations

$$
\begin{aligned}
& \Delta_{\Psi_{\mu}^{a}(x)}(\alpha X+\beta Y)=\alpha \Delta_{\Psi_{\mu}^{a}(x)} X+\beta \Delta_{\Psi_{\mu}^{a}(x)} Y, \\
& \Delta_{\Psi_{\mu}^{a}(x)}(X Y)=X \Delta_{\Psi_{\mu}^{a}(x)} Y+(-1)^{\varepsilon_{X} \varepsilon_{Y}} Y \Delta_{\Psi_{\mu}^{a}(x)} X, \\
& \Delta_{\Psi_{\mu}^{a}(x)}\{X, Y\}=\left\{\Delta_{\Psi_{\mu}^{a}(x)} X, Y\right\}+\left\{X, \Delta_{\Psi_{\mu}^{a}(x)} Y\right\} .
\end{aligned}
$$

We see then that $\Delta_{\Psi_{\mu}^{a}(x)}$ gives rise to a graded derivation with the usual statistics, while the last formula allows us to determine the important result

$$
\int_{1} \cdots \int_{n} \widehat{A}_{1} \cdots \hat{A}_{n} \Delta_{\Psi_{n}} \cdots \Delta_{\Psi_{1}}\{X, Y\}=\sum_{0 \leq m \leq n}\left(\begin{array}{c}
n \\
m
\end{array}\right)\left\{\Delta_{\Psi_{1}} \cdots \Delta_{\Psi_{m}} X, \Delta_{\Psi_{m+1}} \cdots \Delta_{\Psi_{n}} Y\right\}
$$

where we have introduced the shorthand notation $\int_{i}=\int \mathrm{d}^{4} y_{i}, \widehat{A}_{i}=\widehat{A}_{\mu_{i}}^{a_{i}}\left(y_{i}\right)$ and $\Psi_{i}=\Psi_{\mu_{i}}^{a_{i}}\left(y_{i}\right)$.

From the operator $\Delta_{\Psi}$ one can then define a mapping $E_{\Psi}$, given in terms of a formal power series in the background field $\widehat{A}$ as follows

$$
\begin{aligned}
\Phi(x) & =E_{\Psi}(\phi(x)) \\
& \equiv \sum_{n \geq 0} \frac{1}{n !} \int_{1} \cdots \int_{n} \widehat{A}_{1} \cdots \widehat{A}_{n}\left[\Delta_{\Psi_{n}} \cdots \Delta_{\Psi_{1}} \phi(x)\right]_{\widehat{A}=0},
\end{aligned}
$$


with an identical expansion holding for the antifields variables. Then Eq. (2.6) constitutes the sought for canonical mapping between the old and the new variables.

The canonicity property is a direct consequence of Eq. (2.5) above, since the latter directly implies the identity $E_{\Psi}\{X, Y\}=\left\{E_{\Psi} X, E_{\Psi} Y\right\}$. On the other hand, to see that the new variables are indeed solutions of Eqs. (2.4), let us consider first the case of a bosonic field $\Phi$ and expand both the latter and the fermionic generator $\Psi_{\mu}^{a}$ in power series w.r.t. the background field $\widehat{A}$. Schematically, one has

$$
\begin{gathered}
\Phi=\phi+\sum_{n \geq 0} \frac{1}{n !} \int_{1} \cdots \int_{n} \widehat{A}_{1} \cdots \widehat{A}_{n} \Phi_{1 \cdots n}, \\
\Psi_{0}=\psi_{0}+\sum_{n \geq 0} \frac{1}{n !} \int_{1} \cdots \int_{n} \widehat{A}_{1} \cdots \widehat{A}_{n} \Psi_{01 \cdots n},
\end{gathered}
$$

and finds, up to third order in $\widehat{A}$

$$
\begin{aligned}
& \left.\Delta \Psi_{1} \phi\right|_{\widehat{A}=0}=\left\{\phi, \psi_{1}\right\}, \\
& \left.\Delta \Psi_{2} \Delta \Psi_{1} \phi\right|_{\widehat{A}=0}=\left\{\left\{\phi, \psi_{1}\right\}, \psi_{2}\right\}+\left\{\phi, \Psi_{12}\right\}, \\
& \left.\Delta \Psi_{3} \Delta \Psi_{2} \Delta \Psi_{1} \phi\right|_{\widehat{A}=0}=\left\{\left\{\left\{\phi, \psi_{1}\right\}, \psi_{2}\right\}, \psi_{3}\right\}+\left\{\phi, \Psi_{123}\right\} \\
& +\left\{\left\{\phi, \Psi_{12}\right\}, \psi_{3}\right\}+\left\{\left\{\phi, \Psi_{23}\right\}, \psi_{1}\right\}+\left\{\left\{\phi, \Psi_{31}\right\}, \psi_{2}\right\},
\end{aligned}
$$

where in the last equation we have symmetrized all indices, and used the (graded) Jacobi identity together with the result $\int_{1} \int_{2} \widehat{A}_{1} \widehat{A}_{2} \frac{\delta}{\delta \widehat{A}_{3}}\left\{\Psi_{1}, \Psi_{2}\right\}=0$. It can then be checked that the above terms are indeed the solutions (up to third order in $\widehat{A}$ ) of the first of Eqs. (2.4). The fermionic case, e.g., a fermionic antifield $\Phi^{*}$, can be treated in the same way.

Let us end by observing that there is a close relation with the theory of Lie transforms in classical analytical mechanics. Suppose that one wants to find out the finite canonical transformation, depending on the small parameter $\varepsilon$, which maps the canonically conjugated variables $(q, p)$ into the new ones $(Q, P)$, under the assumption that $Q, P$ satisfy the following differential equations:

$$
\frac{\mathrm{d} Q}{\mathrm{~d} \varepsilon}=\frac{\partial}{\partial P} V(p, q ; \varepsilon) ; \quad \frac{\mathrm{d} P}{\mathrm{~d} \varepsilon}=-\frac{\partial}{\partial Q} V(p, q ; \varepsilon) .
$$

The generating functional $V(p, q ; \varepsilon)$ of the canonical transformations depends on a small parameter $\varepsilon$. Then the solution is obtained by a Lie series of the operator $\Delta_{V}=\{\cdot, V\}+\frac{\partial}{\partial \varepsilon}$, where the bracket is here the usual Poisson bracket [13].

\section{Lattice background field method}

On the lattice, the background gauge-fixing can be implemented by minimizing the functional $[13,15]$

$$
F[g]=-\int \mathrm{d}^{4} x \operatorname{Tr}\left(A_{\mu}^{g}-\hat{A}_{\mu}\right)^{2}
$$

w.r.t. the group element $g . A_{\mu}^{g}$ is the gauge transform of the gauge field $A_{\mu}$. After minimizing $F[g]$, one gets the background Landau gauge condition $\widehat{\mathscr{D}}_{\mu}\left(A_{\mu}^{g}-\hat{A}_{\mu}\right)=0$. The canonical transformation, 
reconstructing the dependence on the background field, is the one induced by the mapping $A_{\mu}^{\prime}=$ $A_{\mu}^{g(A, \hat{A})}-\hat{A}_{\mu}$, where the new gauge field $A_{\mu}^{\prime}$ obeys the Landau gauge condition.

If, as a consequence of the existence of the Gribov copies, multiple minima are present, they will be parameterized by different functions $g_{i}(A, \hat{A})$. Each of them controls in principle the dependence on the background, provided that one restrict himself to the region of validity of each $g_{i}$.

\section{Conclusions}

We have reviewed the approach to the implementation of the BFM based on canonical transformations proposed in [13]. This approach has a very general range of applicability: it encompasses the standard perturbative treatment of the BFM and provides a way to extend the BFM in a systematic way to non-perturbative settings. In the lattice case, it can in principle disentangle the solutions related to different Gribov copies, thus allowing to obtain the canonical mapping which governs the dependence on the background field around each of the minima of the gauge-fixing functional. In addition, notice that the comparison between the continuum and the lattice computations might be greatly eased by the powerful relations between 1-PI background and quantum amplitudes, stemming from the background gauge invariance.

Finally, our approach could also be applied to non-perturbative frameworks in the continuum, like the 2-PI formalism and the Schwinger-Dyson equations.

\section{References}

[1] For a review see e.g. T. Schafer and E. V. Shuryak, Rev. Mod. Phys. 70 (1998) 323.

[2] G. 't Hooft, Phys. Rev. D 14 (1976) 3432 [Erratum-ibid. D 18 (1978) 2199].

[3] F. Meier and H. Walliser, Phys. Rept. 289 (1997) 383.

[4] L. F. Abbott, Acta Phys. Polon. B13 (1982) 33; Nucl. Phys. B 185 (1981) 189.

[5] S. Ichinose and M. Omote, Nucl. Phys. B 203 (1982) 221; D. M. Capper and A. MacLean, Nucl. Phys. B 203 (1982) 413.

[6] A. Denner, G. Weiglein and S. Dittmaier, Nucl. Phys. B 440 (1995) 95; P. A. Grassi, T. Hurth and M. Steinhauser, Nucl. Phys. B 610 (2001) 215.

[7] S. J. Gates, M. T. Grisaru, M. Rocek et al., Front. Phys. 58 (1983) 1.

[8] M. Luscher and P. Weisz, Nucl. Phys. B 452 (1995) 213.

[9] H. Neuberger, Phys. Lett. B 183 (1987) 337.

[10] P. A. Grassi, Nucl. Phys. B 462 (1996) 524; C. Becchi and R. Collina, Nucl. Phys. B 562 (1999) 412;

R. Ferrari, M. Picariello and A. Quadri, Annals Phys. 294 (2001) 165.

[11] C. Becchi, "Introduction to BRS symmetry," hep-th/9607181.

[12] D. Binosi and A. Quadri, Phys. Rev. D 85 (2012) 085020.

[13] D. Binosi and A. Quadri, "The Background Field Method as a Canonical Transformation," arXiv:1203.6637 [hep-th]. To appear in Phys. Rev. D - Rapid communications.

[14] D. Binosi and A. Quadri, Phys. Rev. D 84 (2011) 065017.

[15] A. Cucchieri and T. Mendes, arXiv:1204.0216 [hep-lat]. 